\section{IN BRIEF}

\section{$\Rightarrow$ EPIGENETICS}

Transcriptional silencing of Polo-like kinase 2 (SNKIPLK2) is a frequent event in B-cell malignancies.

Syed, N. et al. Blood 107, 250-256 (2006)

The Polo-like kinases (PLKs) regulate cell-cycle and DNAdamage-induced checkpoints, but their function as tumour suppressors in human cancers has not been confirmed. This paper demonstrates that PLK2 is transcriptionally downregulated in B-cell malignancies owing to the aberrant methylation of a CpG island located in the $5^{\prime}$ end of PLK2. Restoration of PLK2 expression in these cells induced apoptosis, indicating why expression of this gene is selected against.

\section{SIGNALLING}

A role for the scaffolding adapter GAB2 in breast cancer.

Bentires-Alj, M. et al. Nature Med. 12, 114-121 (2006)

The transduction of many extracellular signals is enhanced by scaffolding adaptors. The gene that encodes one adaptor, GRB2-associated binding protein 2 (GAB2), maps to 11q13-14, a region that is commonly amplified in breast cancer. The authors show that overexpression of GAB2 induces proliferation of nontransformed mammary cell lines and increases tumour growth in ERBB2 mouse models of breast cancer. Therefore, GAB2 overexpression might be an important consequence of 11q13 amplification in human breast cancers.

\section{$\Rightarrow$ STEM CELLS}

Decatenation checkpoint deficiency in stem and progenitor cells.

Damelin, M., Sun, Y. E., Sodja, V. B. and Bestor, T. H. Cancer Cel/ 8 479-484 (2005)

The decatenation checkpoint delays entry into mitosis until chromosomes have been disentangled. Tim Bestor and colleagues have evidence that the efficiency of this checkpoint is reduced in undifferentiated cells such as stem cells. They show that embryonic stem cells often complete cell division with entangled chromosomes, resulting in aneuploid daughter cells. This deficiency probably increases the rate of chromosome aberrations in cancer stem cells.

\section{BIOMARKERS}

Preoperative serum tissue factor levels are an independent prognostic factor in patients with ovarian carcinoma.

Han, L. Y. et al. J. Clin. Oncol. 27 Dec 2005 (doi:10.1200/ JCO.2005.02.9181)

Tissue factor, a blood coagulant, is implicated in regulating tumour angiogenesis. Han et al. examined whether preoperative levels of tissue factor could serve as a prognostic marker in ovarian cancer patients. They assayed tissue-factor serum levels in 98 women with invasive epithelial carcinoma, 30 with low-malignant-potential tumours and 16 with benign tumours. A validation group of 39 women with metastatic ovarian cancer was also analysed. The results show that tissuefactor serum levels $\geq 190 \mathrm{pg} \mathrm{ml}^{-1}$ are significantly associated with decreased patient survival and that an increased pre-operative tissue-factor serum level is an independent prognostic factor for death from disease.

\title{
Marked aggression
}

Gene-expression profiling is an important tool for classifying cancers into subgroups with prognostic significance. However, not much is known about how most of the individual genes in these signatures contribute to their associated clinical phenotypes. Jose Moyano et al. have linked $\alpha$-basic crystallin ( $\alpha \mathrm{B}$-crystallin), a stress-response protein with oncogenic potential, to an aggressive subtype of human breast tumours (basal-like) identified by gene profiling.

The basal-like group of breast tumours is oestrogen receptornegative and ERBB2-negative, and is associated with short overall and relapse-free survival times, but little is known about how the genes expressed by this tumour type lead to its aggressive behaviour. Moyano et al. examined existing breast cancer microarray data and noticed that $\alpha \mathrm{B}$-crystallin, a cell stress protein of long-standing interest to their laboratory, was frequently expressed in this tumour type.

$\alpha \mathrm{B}$-crystallin is a member of the small-heat-shock-protein (HSP) family. These proteins function as stress-induced molecular chaperones that promote cell survival. Ectopic expression of $\alpha \mathrm{B}$-crystallin protects cells against a broad range of apoptotic stimuli, whereas silencing of its expression with RNA interference (RNAi) sensitizes cells to apoptosis. $\alpha \mathrm{B}$-crystallin is also highly expressed in gliomas, prostate tumours and renal-cell carcinoma, so Moyano et al. explored its potential function in basal-like breast tumours.

They observed that $\alpha \mathrm{B}$-crystallin was expressed in about half of basal-like breast tumour samples, and its expression could predict shorter survival times in patients independently of other established markers. Overexpression of the wild-type protein, but not a pseudophosphorylation mutant, induced neoplastic changes in mammary acini growth in threedimensional culture systems and transformed immortalized human mammary epithelial cells. These cells underwent anchorageindependent proliferation, became highly migratory and invasive in vitro, and formed invasive mammary carcinomas in nude mice. Studies with the pseudophosphorylation mutant indicated that the oncogenic activity of $\alpha \mathrm{B}$-crystallin is negatively regulated by phosphorylation.

How does $\alpha \mathrm{B}$-crystallin transform mammary epithelial cells? The authors found that overexpression of $\alpha B$-crystallin increased levels of total and phosphorylated extracellular-regulated kinase 1 (ERK1)/ERK2 proteins, activating the ERK-MEK (mitogen-activated protein kinase (MAPK) kinase) signalling pathway. This activation is required for transformation - treatment of the cells with MEK inhibitors suppressed their malignant phenotype. As $\alpha \mathrm{B}$ crystallin is a molecular chaperone, the authors propose that it could regulate ERK1/ERK2 stability or phosphorylation, much as HSP90 regulates the activity of the kinases AKT and RAF.

The authors are examining the expression of $\alpha \mathrm{B}$-crystallin in additional breast cancer cohorts to see if its expression level could be a useful marker of prognosis or drug response. Furthermore, these findings indicate that the MEK inhibitors, which are already in clinical trials for the treatment of other cancer types, might be used to treat patients with basal-like breast tumours.

Kristine Novak

ORIGINAL RESEARCH PAPER Moyano, J. F. et al. $\alpha \mathrm{B}$-crystallin is a novel oncoprotein that predicts poor clinical outcome in breast cancer. J. Clin. Invest. 116, 261-270 (2006) 